
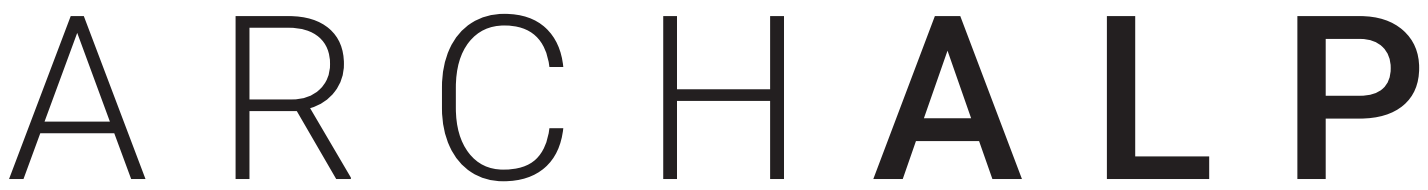

Rivista internazionale di architettura e paesaggio alpino / Revue internationale d'architecture et de paysage dans les Alpes / Internationale Zeitschrift für Alpine Architektur und Landschaft / Revija za alpsko arhitekturo in pokrajino / International journal of alpine architecture and landscape

Rer una nuota abitabilita delle Alpi.

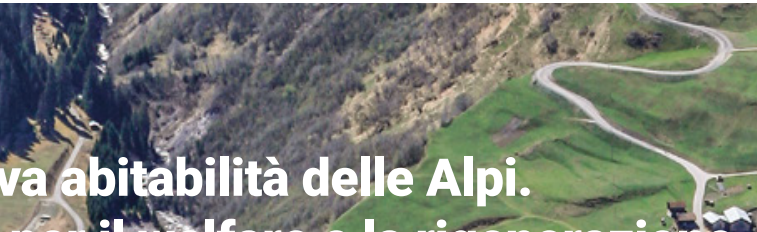
Architeture pet ilf lfare e la rigenerazione For a new inhabifability of the Alps. Architectures for welfare ând regeneration $/$ Pour une nouvelle habitabilité des Alpes. Architectures pour le welfare et la régéhérationy Für eine núue Bewohnbarkeit in den Alpen. Architekturen für Wohf ahrt und Regeneration / Za novo. bivalnost v Alpah, arhitekture za dobrobit in regeneracijo
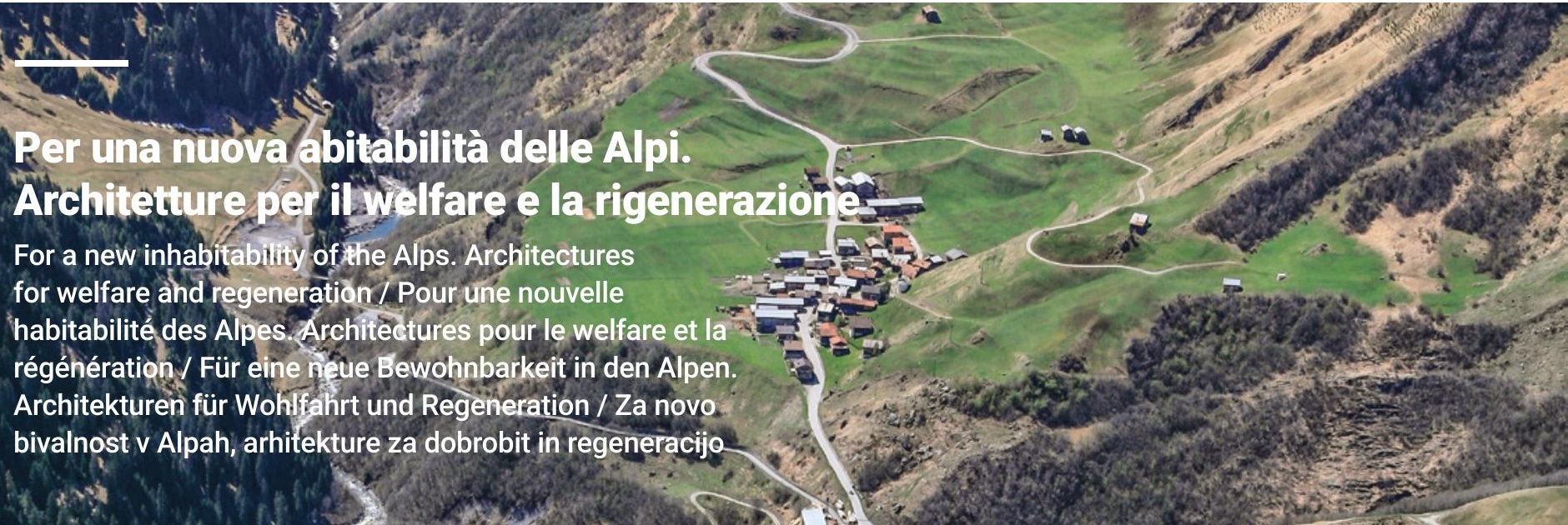
a $x \rightarrow 3$
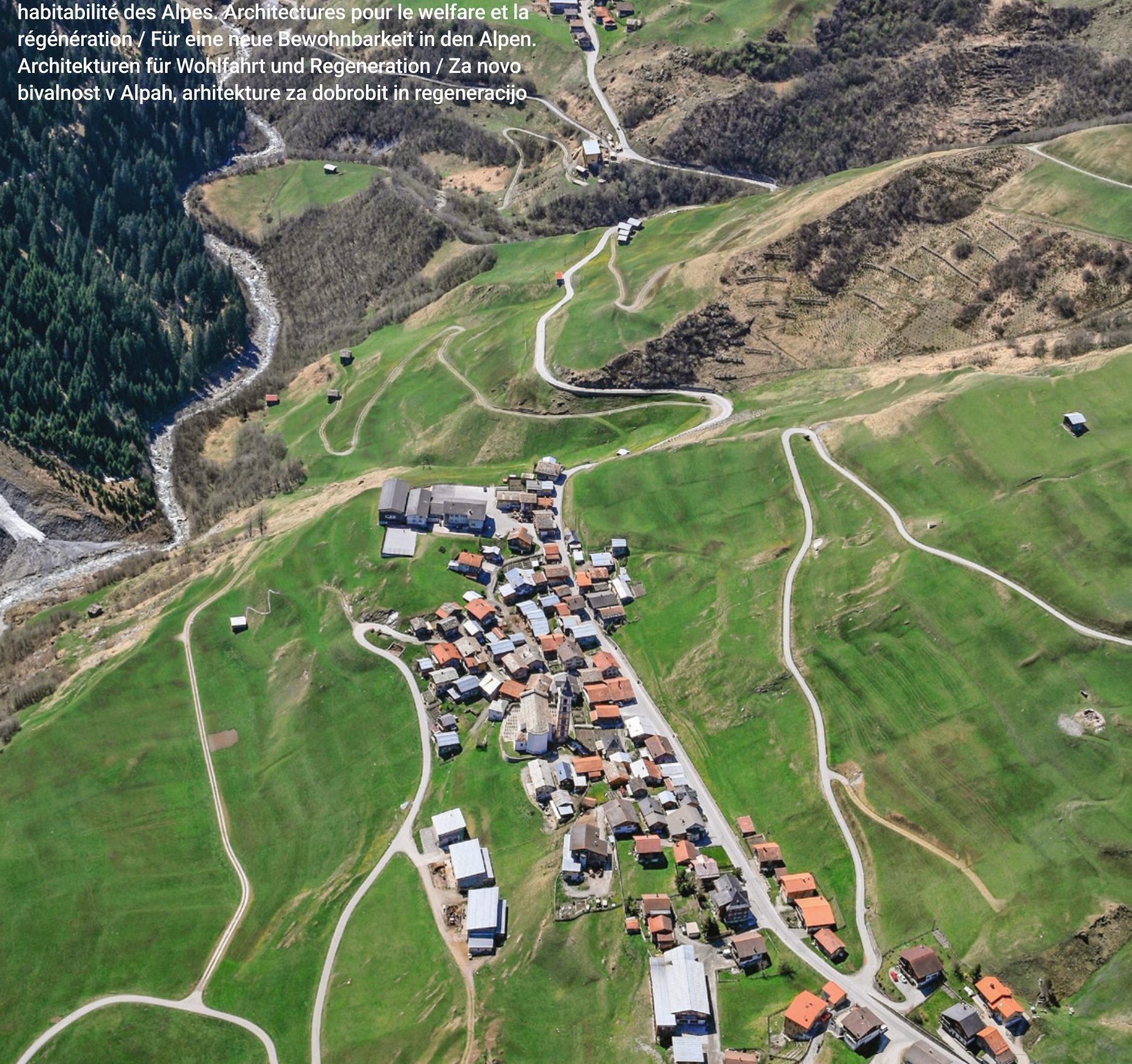


\section{Indice dei contenuti Contents}

\section{Temi}

Sulla centralità di spazio e territorio nel progetto di rigenerazione delle montagne e delle aree interne / On the centrality of space and territory in the project of regeneration of mountains and internal areas Antonio De Rossi, Laura Mascino

I servizi nelle Alpi italiane: quali e dove? Idee per uno scenario post-pandemico / Facilities in the Italian Alps: which ones and where? Ideas for a post-pandemic scenario

Giuseppe Dematteis

Manifesto di Camaldoli per una nuova centralità della montagna

Alla ricerca della distanza perduta. Rigenerare luoghi, persone e immaginari del riabitare alpino / In search of the lost distance. Regenerating places, people and images related to Alpine reinhabitation

Filippo Barbera, Andrea Membretti

Futuro e rigenerazione

Marco Bussone

\section{Esperienze}

Dorferneuerung zwischen Erhalten und Gestalten / 
Valades ousitanes, architettura e rigenerazione / Valades ousitanes,

Ostana e Topolò: hardware, software e welfare nelle comunità di "ritorno" / Ostana and Topolò: hardware, software and welfare in "return" communities

Margherita Valcanover

"Senza mostrare i muscoli": i progetti di rigenerazione di Tao+C e AZL nel solco della "prosperosa società" della Cina contemporanea / "Without flexing one's muscles": the regeneration projects of Tao+C and AZL in the wake of the "prosperous society" of contemporary China Edoardo Bruno, Dalila Tondo

L'archipel Butor. Une régénération, par la culture, d'un village soumis à la métropolisation genevoise / The Butor archipelago. A regeneration, through culture, of a village subject to the metropolisation of Geneva Arnaud Dutheil

Pratiche e progettualità di rigenerazione e welfare: il "Premio triennale Giulio Andreolli - Fare paesaggio" / Actions and projects of regeneration and welfare: the "Premio triennale Giulio Andreolli - Fare paesaggio" Giorgio Tecilla

Architetture e strategie per il welfare. Il caso di Brunico in Val Pusteria / Welfare architectures and strategies. The Bruneck case in Val Pusteria

Eleonora Gabbarini

Arhitektura oživlja / Architecture revives Kristina Dešman, Maja Ivanič

Si Crans-Montana meurt. Soigner le corps malade d'une station / If Crans-Montana dies. Taking care of the ailing body of a tourist resort Patrick Giromini 


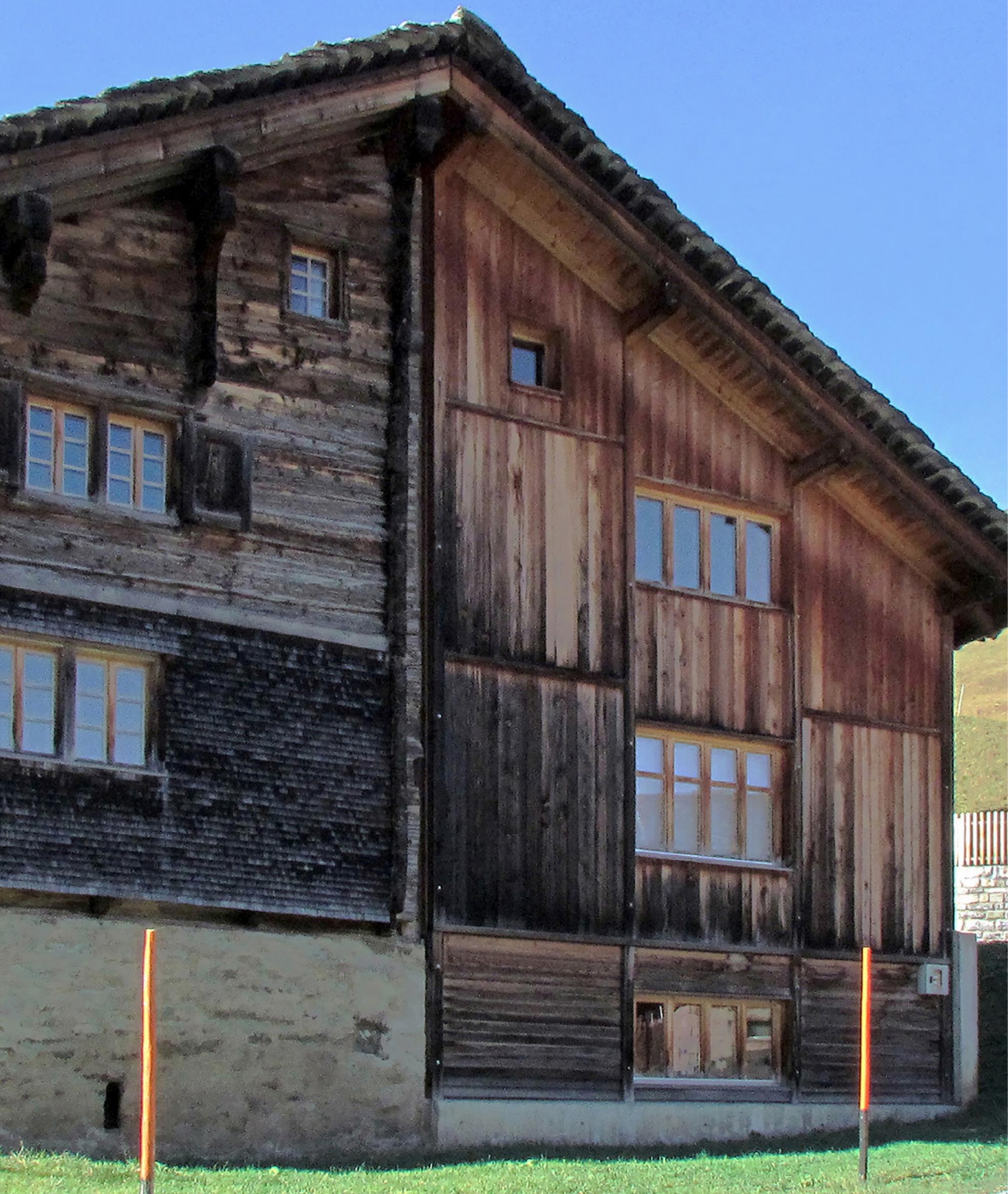

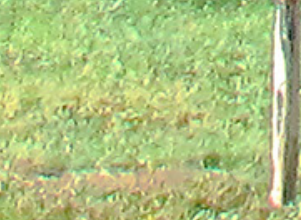

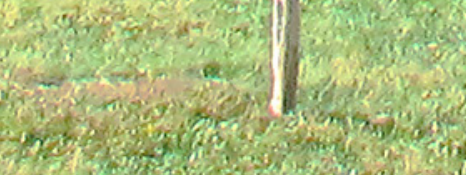

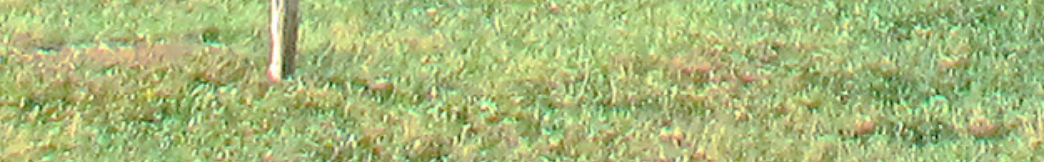




\title{
Marginalità e memoria come valori progettuali nell'esperienza di Gion A. Caminada a Vrin
}

Marginality and memory as planning values in Gion A. Caminada's Vrin experience

\begin{abstract}
The article investigates the regeneration project that was carried out in Vrin, throughout the Alpine context. It deals particularly with architect Gion A. Caminada's modes of action. Caminada had a major role in the Vrin design process in his earlier career. Through the interpretation of the existing anthropic patterns, the architect was able to define some useful spatial basics of contemporary living. Vrin's experience gives us the possibility to discuss some preeminent topics about the reinvention of architectural past elements, that enable us to improve future life.
\end{abstract}

\section{Valerio Botta}

Graduated at the Mendrisio Academy of Architecture, he is both professionally and academically active. He has established collaborations in national and international contexts. He is currently involved in a $\mathrm{PhD}$ program concerning the regeneration of peripheral contexts from the territorial scale to architectural detail.

\section{Keywords}

Ancestral, heritage, memory, marginality, reinvention. 
In apertura Vrin, Casa Comunale, restaurata secondo progetto di Gion

A. Caminada (foto Valerio Botta).
L'area alpina è rappresentativa di un potenziale socio-lavorativo messo in evidenza anche dai suoi villaggi più antichi, che sembrano aver conservato una certa capacità di determinare il paesaggio. D'altra parte molti contesti periferici sono emblematici di soluzioni insediative che ancora oggi potrebbero rappresentare una valida alternativa alla quotidianità urbana (Carlow, 2016). Alcuni agglomerati alpini di origine rurale sono infatti espressione di una strategia insediativa che, oltre a regolare $\mathrm{i}$ rapporti spaziali interni ai nuclei, era orientata alla valorizzazione del territorio in ambito relazionale $\mathrm{e}$ produttivo. Quest'ultimo aspetto veniva gestito tramite il disegno dello spazio aperto, che si estendeva fino ai boschi e agli alpeggi, luogo del lavoro contadino per eccellenza (Camanni, 2017). In questi ambiti l'implementazione infrastrutturale è un'attività quantomai delicata poiché, da un lato, potrebbe compromettere le funzionalità esistenti e dall'altro, se ben articolata, potrebbe servire da propulsore

$\overline{1}$ 
per le attività lavorative consolidate. Infatti, quando l'interesse verso la preesistenza viene coadiuvato da adeguamenti infrastrutturali su quest'ultima, possono crearsi le premesse per un processo di sviluppo sostenibile (Pia, 2019). Le criticità operative in tal senso non riguardano tanto la necessità di ammodernamento di strutture arcaiche, quanto le modalità tramite cui tale ammodernamento viene compiuto. A seconda, infatti, di come una serie di nuove facilities viene introdotto nella preesistenza si possono avere reazioni differenti dal contesto spaziale.

La Svizzera, paese quasi interamente alpino, è una zona fortemente interessata dal dibattito sviluppatosi attorno alla rigenerazione di contesti periferici di origine rurale. In particolare, la Val Lumnezia, area interna alla Surselva, è stata scenario di un esperimento progettuale sulla preesistenza che ha coinvolto diversi ambiti disciplinari. Le princi-

Fig. 1

Vrin, planimetria (disegno Valerio Botta) nicipalità per formare il comune unico di Lumnezia. Questo comune verso la fine degli anni Settanta venne toccato dal fenomeno dello spopolamento, in seguito alla migrazione di alcuni suoi abitanti. Un fenomeno quest'ultimo dovuto in parte alla diminuzione del lavoro disponibile nel settore agropastorale, divenuto sempre più meccanizzato. Il villaggio di Vrin, il più esteso tra i villaggi che componevano il comune, tra gli anni Cinquanta e gli anni Novanta aveva quasi dimezzato la sua popolazione (Schlorhaufer, 2005).

In risposta a tali dinamiche, verso la fine degli anni Ottanta, diverse organizzazioni avviarono un processo di rivitalizzazione volto a preservare il tessuto antropico esistente, adeguandolo in funzione delle generazioni future. Tale processo era spinto dall'obiettivo di valorizzare, tramite operazioni urbanistiche ed architettoniche, alcuni settori produttivi già attivi all'interno del comune.

Le associazioni coinvolte in questo progetto toccavano diversi aspetti riguardanti l'ambito della rige-

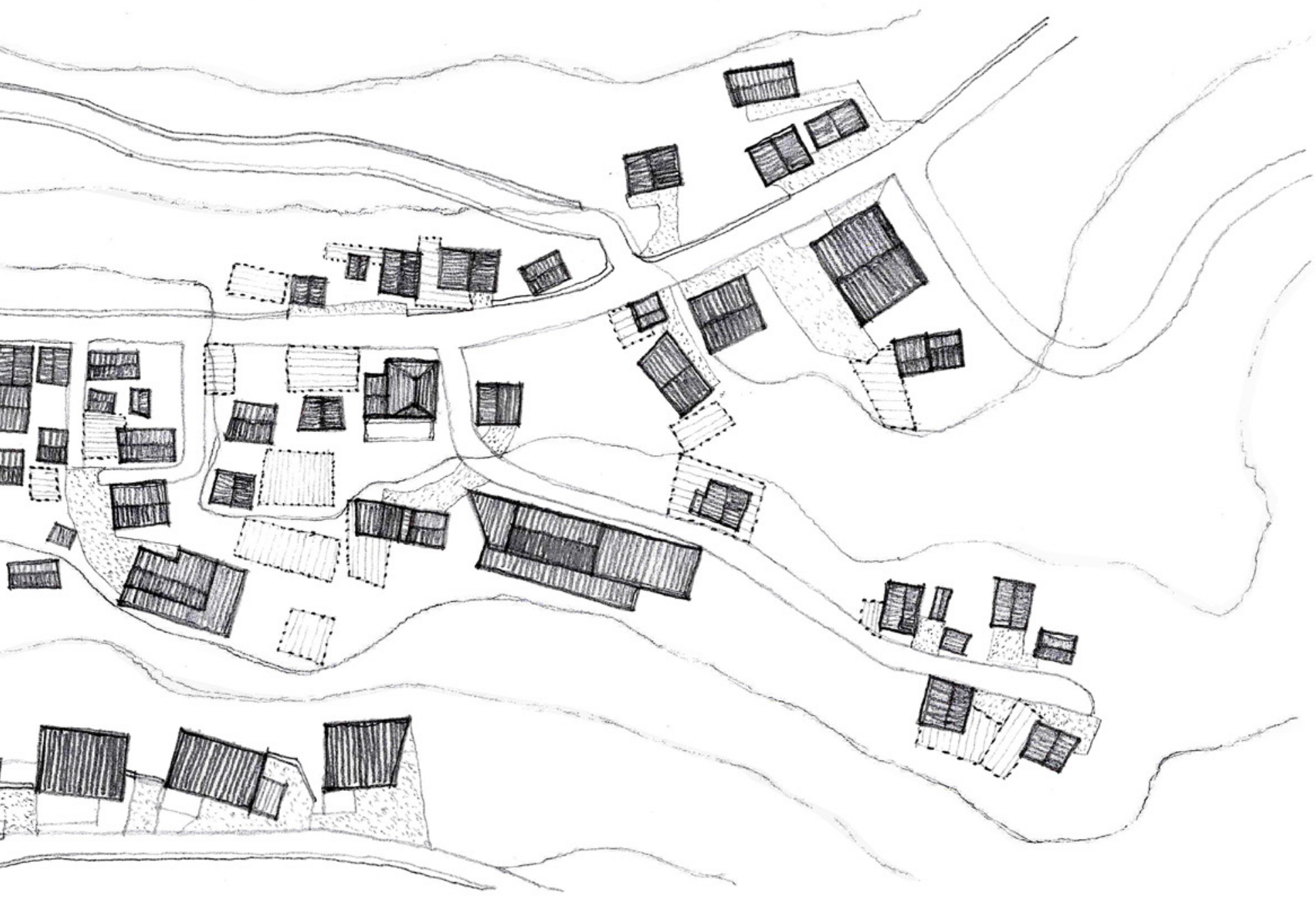


nerazione, coinvolgendo sia il settore pubblico che quello privato. Tra queste vi erano: la fondazione di architetti Pro Vrin, nata nel 1979, la Soprintendenza per i beni culturali del Cantone, il Consorzio per il miglioramento fondiario e l'autorità comunale. Alla Pro Vrin, in particolare, si deve la definizione di alcuni principi insediativi che sarebbero serviti come linee guida in fase di realizzazione del progetto. Tra i membri della fondazione c'era il giovane architetto Gion A. Caminada, abitante della valle, che ebbe un ruolo fondamentale per lo sviluppo del processo rigenerativo. Instaurando un dialogo con gli abitanti di Vrin, Caminada sensibilizzò la comunità locale a riguardo di come l'architettura potesse favorire gli ambiti lavorativi e relazionali (Schlorhaufer, 2005). Allo stesso tempo l'architetto raccolse le richieste della collettività tramite interviste, conversazioni interpersonali e riunioni, ponendole successivamente in dibattito all'interno delle varie associazioni coinvolte nel progetto. Una volta discusso su cosa intervenire il processo di rigenerazione si concretizzò attraverso azioni sul campo.

La prima grande operazione attuata dalle associazioni può farci capire quanto il progetto si estendesse oltre le dinamiche spaziali dei nuclei costruiti, fino a interessare questioni territoriali e produttive. All'inizio degli anni Novanta, infatti, furono avviate una serie di attività a sostegno del lavoro agropastorale, come la costruzione di alpeggi e strade per facilitare l'accesso ai campi. In quest'ottica, grazie alla collaborazione tra Caminada e il Consorzio per il miglioramento fondiario, vennero raggruppate le parcelle aventi la stessa proprietà, ma situate in aree della valle distanti tra loro. Quest'ultima operazione seguiva le direttive di un piano di rilottizzazione, redatto proprio da Caminada e finalizzato a migliorare l'accessibilità dei terreni disponibili, minimizzando la costruzione di strade di servizio (Schlorhaufer, 2005).

Ad arricchire questa fase del processo di rigenerazione ci fu la progettazione e la realizzazione di alcuni fabbricati, funzionali alla formazione di un consorzio agrario all'interno del villaggio di Vrin. Caminada fece destinare per questo nuovo apparato produttivo un'area presso il confine più a valle del nucleo, in modo da ridefinire, tramite tre edifici, due stalle con fienile e un mattatoio, il limite tra paesaggio costruito e paesaggio naturale.

La prima fase del processo di rigenerazione evidenziava il ruolo preminente attribuito al progetto dello spazio aperto. Centralità mantenuta anche nelle fasi di pianificazione successive, riguardanti la ridefinizione dello spazio pubblico all'interno dei villaggi, il restauro di alcuni edifici e la realizzazione di nuove strutture private e collettive. Tale fase del progetto sembra esser stata caratterizzata dal ten- tativo di valorizzare il principio dello Zwischenraum, di norma riguardante l'interno degli edifici, ma in qualche modo estensibile anche al tema del vuoto pubblico. Lo Zwischenraum, alla lettera "spazio tra", è l'area d'intermediazione che si interpone tra forme costruite. È uno spazio di transizione e allo stesso tempo un vuoto definito, in cui la vita si compie nella sua complessità quotidiana (Kepstein, 2015). Esso accomuna differenti agglomerati nel territorio alpino, in cui è spesso formato tramite fabbricati autonomi posti in prossimità tra loro. Nel villaggio di Vrin, questo tipo di spazio, è espresso dalla globalità delle aree pubbliche aperte. Queste messe in giustapposizione tra loro, rendono il nucleo permeabile e percorribile in tutta la sua estensione. In questo modo lo spazio d'intermediazione di Vrin si configura come zona usufruibile in tutta la sua superficie, legando gli edifici che compongono l'agglomerato.

Nel tentativo di reinterpretare quest'ambito spaziale, Caminada decise di rafforzarne la forma tramite il ridisegno del piano di calpestio e attraverso l'attuazione di alcune norme inserite nel piano regolatore. Tra queste, particolarmente rilevante, è la norma che vieta la delimitazione di aree aperte ad eccezione degli orti, definibili solo tramite recinti permeabili alla vista. Una regola quest'ultima che consente il mantenimento della percezione dello spazio aperto, concessa visivamente quando quest'ultimo non è transitabile.

Inoltre per mantenere l'espressività dello spazio pubblico nei villaggi e per favorire l'attività produttiva riguardante carpenteria e artigianato venne concepita un'ulteriore regola. Quest'ultima obbliga a restaurare o realizzare nuovi edifici utilizzando il materiale del legno. Un materiale che può essere ricavato dai numerosi boschi di pini che caratterizzano la val Lumnezia e che, successivamente, può essere lavorato da aziende locali. In questo modo, tramite l'attuazione di una nuova regola, è stato reso possibile il mantenimento di una filiera produttiva e la conservazione di un elemento architettonico già riguardante la definizione volumetrica dei villaggi.

Infine, il processo di reinvenzione e rigenerazione architettonica, che coinvolse Caminada, riguardò la progettazione di nuovi edifici, pubblici e privati. Anche in questo caso lo spazio d'intermediazione pubblico sembra aver avuto un ruolo determinante. Le nuove costruzioni progettate dall'architetto, vennero infatti posizionate all'interno del tessuto in modo da ricucire le aree aperte meno definite del villaggio, ridisegnandole discretamente e creando una continuità spaziale tra passato e presente $(\mathrm{Ca}$ niggia, 1992).

Questa particolare attenzione verso i rapporti instaurati tra le varie volumetrie che definiscono il 


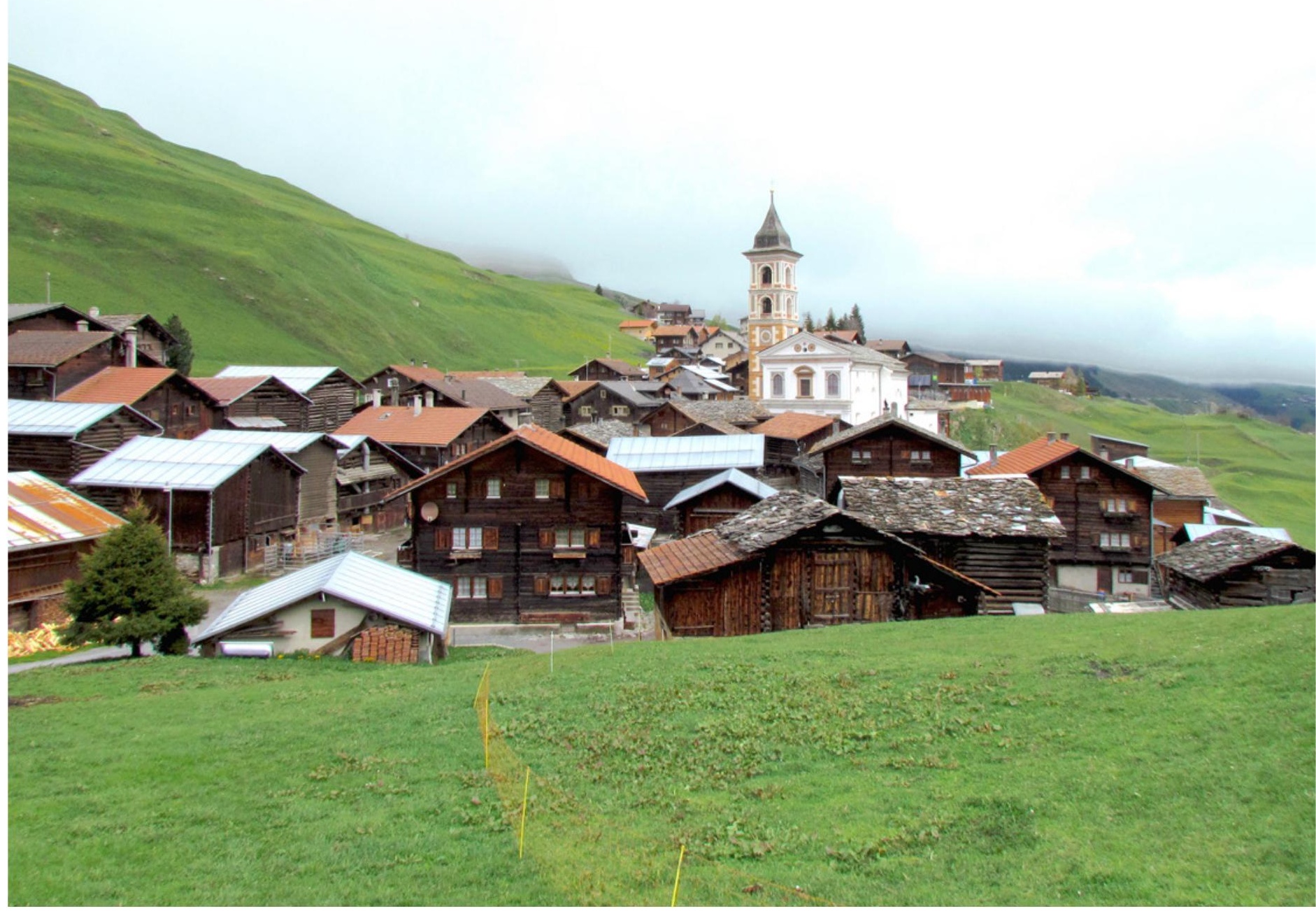

Fig. 2 (foto Valerio Botta). vuoto d'intermediazione è evidente quando si osserva la Stiva da Morts, anch'essa progettata da Caminada. Si tratta di un edificio di servizio pubblico che è andato ad aggiungersi ad altre facilities, di nuova costruzione all'interno del nucleo, pensate da Caminada assieme alla comunità. (Schlorhaufer, 2005). La Stiva da Morts è una struttura che ospita la camera mortuaria dove gli abitanti del villaggio possono dare l'ultimo saluto alle persone decedute. A dispetto della sua funzione l'edificio è concepito come casa della collettività ed esprime tale concetto a partire dal suo posizionamento. La Stiva da Morts si trova, infatti, ai confini dello spazio aperto antistante la chiesa di Vrin, al centro del villaggio. In quest'area l'edificio è accessibile sia dal nucleo che dall'interno del recinto in cui è situato il cimitero, creando una delicata connessione tra area sacra e area profana. Questa sorta d'interdipendenza spaziale è riscontra- bile anche osservando la pianta dell'edificio, caratterizzata da una distribuzione per celle poste in successione tra loro. Tale interconnessione è enfatizzata dalle profonde soglie ricavate tra i vari ambienti. La costruzione, infatti, è stata pensata come una massa scavata, che ospita alcune nicchie in affaccio sulle aree comuni e delle forature che proiettano lo sguardo verso l'esterno. Questa forma è ottenuta attraverso la manipolazione della struttura in legno, realizzata tramite la tecnica dello strick-bau. Quest'ultimo è un ulteriore aspetto della reinvenzione attuata dal progettista, che, in questo caso, riguarda una tecnica costruttiva tradizionale ampiamente sviluppata nel contesto dei Grigioni (Aspesi, Cataldi, 2013).

Il tema del vuoto come spazio d'intermediazione è stato però approfondito da Caminada anche con tecniche costruttive che impiegano materiali differenti dal legno e in contesti distanti da Vrin. Em- 


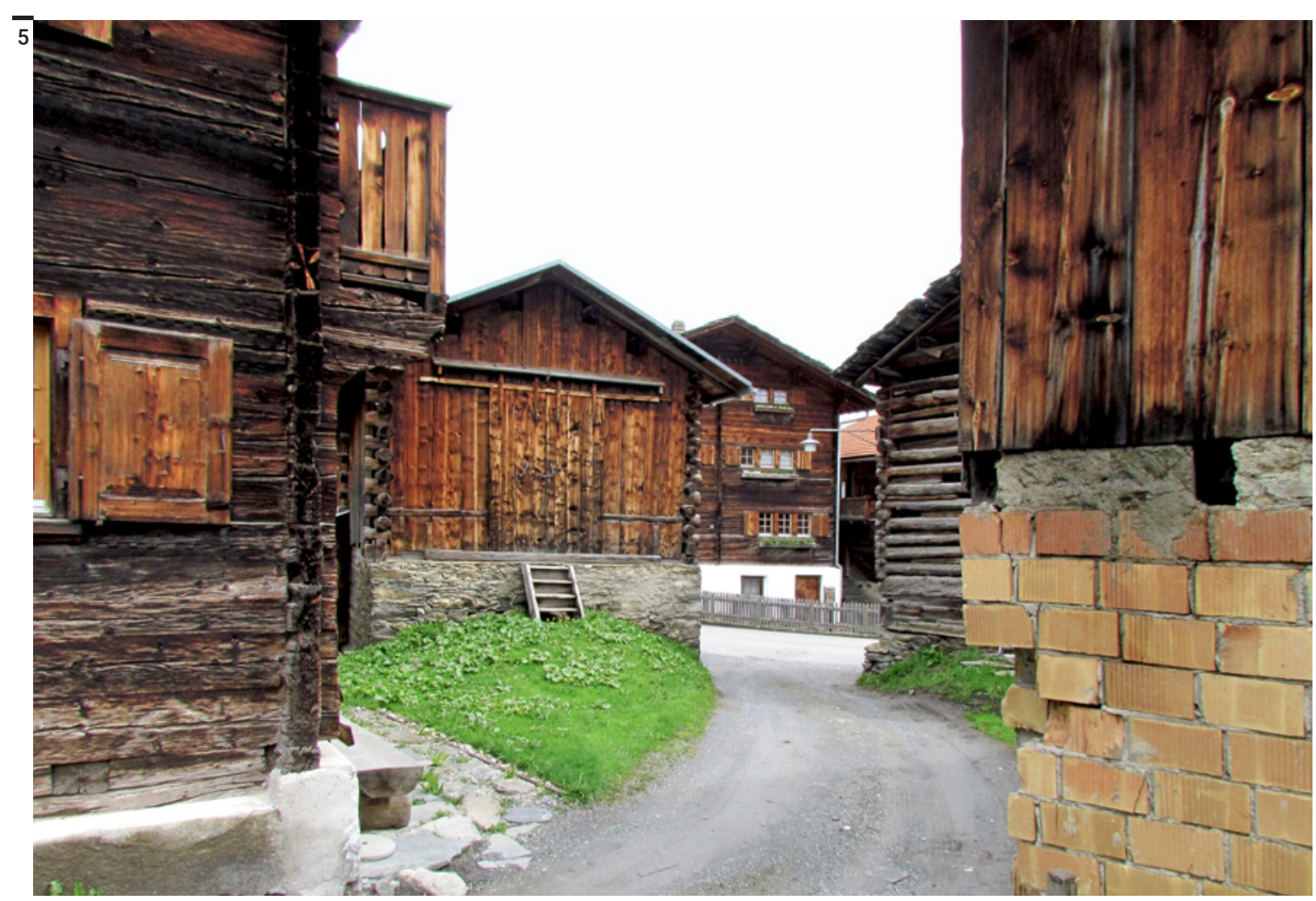

blematico in tal senso è il progetto di un dormitorio per ragazze a Disentis (Schlorhaufer, 2005), dove lo spazio intermedio si sviluppa come area filtro comune, interposta tra i locali delle camere e il vano scala. Quest'ultimo si configura come elemento di connessione verticale in calcestruzzo, in cui sono ricavate delle nicchie per permettere alle abitanti del dormitorio di sostare per riposare e socializzare.

Fig. 5 La riproposizione del tema dello spazio intermeVrin. Lo spazio d'intermediazione tra gli edifici (foto Valerio Botta). le, mettendo in luce il valore della reinvenzione in ambiti compositivi di carattere generale (Caniggia, Maffei, 2008).

Osservandoli in quest'ottica, sia il caso progettuale di Vrin, che quello di Disentis, dimostrano come l'esperienza di un luogo possa contribuire alla costruzione di un bagaglio di riferimenti architettonici dal valore trasversale. Un bagaglio emblematico di un'eredità che può essere riproposta nella sua validità contemporanea, mostrandoci alcuni efficaci metodi di antropizzazione (Carlow, 2016).

\section{Bibliografia}

Aspesi G. Mario, Cataldi Giancarlo (2013), Casa alpina in tronchi / Blockbau. Varianti locali ed evoluzione tipologica, Priuli e Verlucca Editori, Scarmagno.

Camanni Enrico (2017), Storia delle Alpi. Le più belle montagne del mondo raccontate, Edizioni Biblioteca dell'Immagine, Pordenone.

Caniggia Gianfranco (1976), Strutture dello Spazio Antropico. Studi e note, (ed. 1992) Alinea, Firenze. Caniggia Gianfranco, Maffei Gian Luigi (1979), Lettura dell'edilizia di base, (ed. 2008) Alinea, Firenze.

Carlow Vanessa Miriam (a cura di) (2016), Ruralism. The Future of Small Towns and Villages in an Urbanizing World, Jovis Verlag, Berlin.

De Rossi Antonio (a cura di) (2018), Riabitare l'ttalia. Le aree interne tra abbandoni e riconquiste, Donzelli, Roma. Kapstein Glenda (a cura di) (1988), Espacios intermedios. Respuesta arquitectónica al medio ambiente, (ed. 2015) Ediciones Arq, Santiago.

Pia Fiona (2019), Urbanizing the Alps. Densification strategies for mountain villages, Birkhäuser, Basel. Schlorhaufer Bettina (a cura di) (2005), Cul zuffel e l'aura dado. Gion A. Camianda, Quart Verlag, Luzern. 